\title{
An Extension to the Owa-Srivastava Fractional Operator with Applications to Parabolic Starlike and Uniformly Convex Functions
}

\section{Oqlah Al-Refai and Maslina Darus}

School of Mathematical Sciences, Faculty of Science and Technology,

University Kebangsaan Malaysia, Bangi 43600, Selangor, Malaysia

Correspondence should be addressed to Maslina Darus, maslina@ukm.my

Received 8 October 2008; Accepted 6 January 2009

Recommended by Shaher Momani

Let $\mathcal{A}$ be the class of analytic functions in the open unit disk $\mathbb{U}$. We define $\Theta^{\alpha, \beta}: \mathcal{A} \rightarrow \mathcal{A}$ by $\left(\Theta^{\alpha, \beta} f\right)(z):=\Gamma(2-\alpha) z^{\alpha} D_{z}^{\alpha}\left(\Gamma(2-\beta) z^{\beta} D_{z}^{\beta} f(z)\right),(\alpha, \beta \neq 2,3,4 \ldots)$, where $D_{z}^{\gamma} f$ is the fractional derivative of $f$ of order $\gamma$. If $\alpha, \beta \in[0,1]$, then a function $f$ in $\mathcal{A}$ is said to be in the class $\operatorname{SP}_{\alpha, \beta}$ if $\Theta^{\alpha, \beta} f$ is a parabolic starlike function. In this paper, several properties and characteristics of the class $\mathrm{SP}_{\alpha, \beta}$ are investigated. These include subordination, characterization and inclusions, growth theorems, distortion theorems, and class-preserving operators. Furthermore, sandwich theorem related to the fractional derivative is proved.

Copyright (C) 2009 O. Al-Refai and M. Darus. This is an open access article distributed under the Creative Commons Attribution License, which permits unrestricted use, distribution, and reproduction in any medium, provided the original work is properly cited.

\section{Introduction and Definitions}

Let $\mathcal{A}$ be the class of functions analytic in the open unit disk $\mathbb{U}:=\{z:|z|<1\}$ and let $\mathscr{A}[a, n]$ be the subclass of $\mathcal{A}$ consisting of functions of the form

$$
g(z)=a+a_{n} z^{n}+a_{n+1} z^{n+1}+\cdots,
$$

and $\mathcal{A}_{0}$ be the class of functions $f$ in $\mathcal{A}$ of the form

$$
f(z)=z+\sum_{n=1}^{\infty} a_{n} z^{n+1} .
$$


Let $\tau$ be the subclass of $\mathcal{A}_{0}$ consisting of functions $f$ of the form

$$
f(z)=z-\sum_{n=1}^{\infty} a_{n} z^{n+1}, \quad\left(a_{n} \geq 0\right) .
$$

A function $f$ in $A_{0}$ is said to be uniformly convex in $\mathbb{U}$ if $f$ is a univalent convex function along with the property that, for every circular arc $\gamma$ contained in $\mathbb{U}$, with center $\gamma$ also in $\mathbb{U}$, the image curve $f(\gamma)$ is a convex arc. The class of uniformly convex functions is denoted by UCV (for details, see [1]). It is well known from [2,3] that

$$
f \in \mathrm{UCV} \Longleftrightarrow\left|\frac{z f^{\prime \prime}(z)}{f^{\prime}(z)}\right|<\operatorname{Re}\left\{1+\frac{z f^{\prime \prime}(z)}{f^{\prime}(z)}\right\}, \quad(z \in \mathbb{U})
$$

Condition (1.4) implies that

$$
1+\frac{z f^{\prime \prime}(z)}{f^{\prime}(z)}
$$

lies in the interior of the parabolic region

$$
\mathcal{R}:=\left\{w: w=u+i v, v^{2}<2 u-1\right\},
$$

for every value of $z \in \mathbb{U}$. A function $f$ in $\mathcal{A}_{0}$ is said to be in the class of parabolic starlike functions, denoted by SP (cf. [3]), if

$$
\frac{z f^{\prime}(z)}{f(z)} \in \mathcal{R}, \quad(z \in \mathbb{U})
$$

Let the function $\varphi(a, b ; z)$ be given by

$$
\varphi(a, b ; z):=\sum_{n=0}^{\infty} \frac{(a)_{n}}{(b)_{n}} z^{n+1} \quad(b \neq 0,-1,-2, \ldots ; z \in \mathbb{U})
$$

where $(x)_{n}$ is the Pochhammer symbol defined by

$$
(x)_{n}:=\frac{\Gamma(x+n)}{\Gamma(x)}= \begin{cases}1, & n=0 \\ x(x+1)(x+2) \cdots(x+n-1), & n \in \mathbb{N}:=\{1,2,3, \ldots\}\end{cases}
$$

Further, let (cf. [4, 5])

$$
L(a, b) f(z)=\varphi(a, b ; z) * f(z) \quad(f \in \mathcal{A}) .
$$


In terms of Hadamard product or convolution, note that $L(a, a)$ is the identity operator and

$$
L(a, c)=L(a, b) L(b, c) \quad(b, c \neq 0,-1,-2, \ldots) .
$$

It is well known that if $b>a>0$, then $L$ maps $\mathcal{A}$ into itself. We also need the following definitions of a fractional derivative.

Definition 1.1 (cf. $[5,6]$, see also $[7,8]$ ). Let the function $f(z)$ be analytic in a simply connected domain of the $z$-plane containing the origin. The fractional derivative of $f$ of order $\alpha$ is defined by

$$
D_{z}^{\alpha} f(z)=\frac{1}{\Gamma(1-\alpha)} \frac{d}{d z} \int_{0}^{z} \frac{f(\xi)}{(z-\xi)^{\alpha}} d \xi, \quad 0 \leq \alpha<1,
$$

where the multiplicity of $(z-\xi)^{-\alpha}$ is removed by requiring $\log (z-\xi)$ to be real when $z-\xi>0$.

Using Definition 1.1 and its known extensions involving fractional derivatives and fractional integrals, Owa and Srivastava [5] introduced the operator $\Omega^{\alpha}: \mathcal{A} \rightarrow \mathcal{A}$ defined by

$$
\begin{aligned}
\Omega^{\alpha} f(z) & =\Gamma(2-\alpha) z^{\alpha} D_{z}^{\alpha} f(z), \quad \alpha \neq 2,3,4, \ldots \\
& =\sum_{n=0}^{\infty} \frac{\Gamma(n+2) \Gamma(2-\alpha)}{\Gamma(n+2-\alpha)} a_{n} z^{n+1} \\
& =\varphi(2,2-\alpha ; z) * f(z) \\
& =L(2,2-\alpha) f(z) .
\end{aligned}
$$

Note that $\Omega^{0} f(z)=f(z)$.

Corresponding to the operator $\Omega^{\alpha}$ defined in (1.13), Srivastava and Mishra [9] studied the class $\mathrm{SP}_{\alpha}(0 \leq \alpha \leq 1)$ of functions $f \in \mathcal{A}_{0}$ satisfying the inequality

$$
\left|\frac{z\left(\Omega^{\alpha} f(z)\right)^{\prime}}{\Omega^{\alpha} f(z)}-1\right|<\operatorname{Re}\left\{\frac{z\left(\Omega^{\alpha} f(z)\right)^{\prime}}{\Omega^{\alpha} f(z)}\right\}, \quad(z \in \mathbb{U})
$$

In Definition 1.2, we generalize the Owa-Srivastava operator defined in (1.13) as follows.

Definition 1.2. Let $f$ be in $\mathcal{A}$. One defines an operator $\Theta^{\alpha, \beta}: \mathcal{A} \rightarrow \mathcal{A}$ by

$$
\left(\Theta^{\alpha, \beta} f\right)(z)=\Gamma(2-\alpha) z^{\alpha} D_{z}^{\alpha}\left(\Gamma(2-\beta) z^{\beta} D_{z}^{\beta} f(z)\right), \quad(\alpha, \beta \neq 2,3,4, \ldots),
$$

where $D_{z}^{\gamma} f$ is the fractional derivative of $f$ of order $\gamma$. 
From Definition 1.2, we note that

$$
\begin{aligned}
\Theta^{\alpha, \beta} f(z) & =\Theta^{\beta, \alpha} f(z), \quad \Theta^{0,0} f(z)=f(z) \\
\Theta^{\alpha, 0} f(z) & =\Theta^{0, \alpha} f(z)=\Omega^{\alpha} f(z), \quad \Theta^{\alpha, 1} f(z)=z\left(\Omega^{\alpha} f(z)\right)^{\prime}, \\
\Theta^{\alpha, \beta} f(z) & =\Gamma(2-\alpha) z^{\alpha} D_{z}^{\alpha}\left(\Gamma(2-\beta) z^{\beta} D_{z}^{\beta} f(z)\right), \quad(\alpha, \beta \neq 2,3,4, \ldots) \\
& =\sum_{n=0}^{\infty} \frac{\Gamma(n+2) \Gamma(2-\alpha)}{\Gamma(n+2-\alpha)} \frac{\Gamma(n+2) \Gamma(2-\beta)}{\Gamma(n+2-\beta)} a_{n} z^{n+1} \\
& =\varphi(2,2-\beta ; z) * \varphi(2,2-\alpha ; z) * f(z) \\
& =\varphi(2,2-\beta ; z) * L(2,2-\alpha) f(z) \\
& =\varphi(2,2-\beta ; z) * \Omega^{\alpha} f(z) \\
& =L(2,2-\beta) \Omega^{\alpha} f(z) \\
& =\Omega^{\beta}\left(\Omega^{\alpha} f(z)\right)=\Omega^{\alpha}\left(\Omega^{\beta} f(z)\right) .
\end{aligned}
$$

In the present paper, we study a class of analytic functions, related to UCV, SP, and $\mathrm{SP}_{\alpha}$, using the operator $\Theta^{\alpha, \beta}$ defined in Definition 1.2.

Definition 1.3. Let $\mathrm{SP}_{\alpha, \beta}$, where $\alpha, \beta \in[0,1]$ be the class of functions $f \in \mathcal{A}_{0}$ satisfying the inequality

$$
\left|\frac{z\left(\Theta^{\alpha, \beta} f(z)\right)^{\prime}}{\Theta^{\alpha, \beta} f(z)}-1\right|<\operatorname{Re}\left\{\frac{z\left(\Theta^{\alpha, \beta} f(z)\right)^{\prime}}{\Theta^{\alpha, \beta} f(z)}\right\}, \quad(z \in \mathbb{U}) .
$$

It follows that

$$
\begin{gathered}
\mathrm{SP}_{\alpha, \beta} \equiv \mathrm{SP}_{\beta, \alpha}, \quad \mathrm{SP}_{\alpha, 0} \equiv \mathrm{SP}_{0, \alpha} \equiv \mathrm{SP}_{\alpha \prime} \\
\mathrm{SP}_{1,0} \equiv \mathrm{SP}_{0,1} \equiv \mathrm{UCV}, \quad \mathrm{SP}_{0,0} \equiv \mathrm{SP} .
\end{gathered}
$$

Remark 1.4. $f(z) \in \mathrm{SP}_{1,1}$ if and only if $z f^{\prime}(z)$ is uniformly convex function.

Using the definition of $\Theta^{\alpha, \beta}$, we start with proving sandwich theorem related to the fractional derivative. Then, we investigate several properties and characteristics of the general class $\mathrm{SP}_{\alpha, \beta}$ using similar techniques to [9]. These include subordination, inclusions and characterization, growth theorems, and class-preserving operators (like the Hadamard product and various integral transforms).

\section{Sandwich Theorem}

In order to prove our sandwich result, we need first to recall the principle of subordination between analytic functions, let the functions $f$ and $F$ be in $\mathcal{A}$. We say that $f$ is subordinate to 
$F$ or $F$ is superordinate to $f$ in $\mathbb{U}$, written as $f \prec F$, if $F$ is univalent in $\mathbb{U}$,

$$
f(0)=F(0), \quad f(\mathbb{U}) \subseteq F(\mathbb{U})
$$

Let $p, h \in \mathcal{A}$ and let $\phi(s, t ; z): \mathbb{C}^{2} \times \mathbb{U} \rightarrow \mathbb{C}$. If $p$ and $\phi\left(p(z), z p^{\prime}(z) ; z\right)$ are univalent and $p$ satisfies the first-order differential superordination,

$$
h(z) \prec \phi\left(p(z), z p^{\prime}(z) ; z\right)
$$

then $p$ is a solution of the differential superordination (2.2). An analytic function $q$ is called a subordination if $q \prec p$ for all $p$ satisfying (2.2). A univalent subordinant $\tilde{q}$ that satisfies $q \prec \tilde{q}$ for all subordinations $q$ of (2.2) is said to be the best subordinant. An analytic function $q$ is said to be dominant if $p \prec q$ for all $p$ satisfying

$$
\phi\left(p(z), z p^{\prime}(z) ; z\right) \prec h(z)
$$

A univalent dominant $\tilde{q}$ that satisfies $\tilde{q} \prec q$ for all dominants $q$ of (2.3) is said to be the best dominant.

We also need the following definition and lemma.

Definition 2.1 (see [10, page 817, Definition 2]). Denoted by $Q$, the set of all functions $f(z)$ that are analytic and injective on $\overline{\mathbb{U}}-E(f)$, where

$$
E(f)=\left\{\zeta \in \partial \mathbb{U}: \lim _{z \rightarrow \zeta} f(z)=\infty\right\}
$$

and are such that $f^{\prime}(\zeta) \neq 0$ for $\zeta \in \partial \mathbb{U}-E(f)$.

Lemma 2.2 (see [11]). Let $q_{1}, q_{2}$ be two nonzero univalent functions in $\mathbb{U}$, and let $\lambda \neq 0, \mu \in \mathbb{C}$. Further assume that $\mathfrak{R}\left[\mu \bar{\lambda} q_{i}(z)\right] \geq 0$ and for $(i=1,2), z q_{i}^{\prime}(z) / q_{i}(z)$ is starlike univalent in U. If $g \in \mathcal{A}_{0}, z^{k} g^{(k)}(z) / g^{(k-1)}(z) \in \mathcal{A}[1,1] \cap Q\left(k \in \mathbb{N}, g^{(k)}\right.$ is the $k$ th derivative of $\left.g\right)$ and $z g^{(k+1)}(z) / g^{(k)}(z)+\left(\mu z^{k} / \lambda-z\right) g^{(k)}(z) / g^{(k-1)}(z)+k$ is univalent in $\mathbb{U}$, then

$$
\mu q_{1}(z)+\lambda \frac{z q_{1}^{\prime}(z)}{q_{1}(z)}<\lambda\left(\frac{z g^{(k+1)}(z)}{g^{(k)}(z)}+\frac{\left(\mu z^{k} / \lambda-z\right) g^{(k)}(z)}{g^{(k-1)}(z)}+k\right) \prec \mu q_{2}(z)+\lambda \frac{z q_{2}^{\prime}(z)}{q_{2}(z)}
$$

implies

$$
q_{1}(z) \prec \frac{z^{k} g^{(k)}(z)}{g^{(k-1)}(z)} \prec q_{2}(z)
$$

and $q_{1}, q_{2}$ are, respectively, the best subordinant and the best dominant. 
As an application of Lemma 2.2, we prove the following theorem.

Theorem 2.3. Let $q_{1}, q_{2}$ be two nonzero univalent functions in $\mathbb{U}$, and let $k \in \mathbb{N}, \alpha \neq 2,3,4, \ldots$, and $0<\beta<1$. Further, assume that $z q_{1}^{\prime}(z) / q_{1}(z)$ and $z q_{2}^{\prime}(z) / q_{2}(z)$ are starlike univalent in $\mathbb{U}$. If $f \in \mathcal{A}_{0}$,

$$
\begin{aligned}
& \Phi(\alpha, \beta, k ; z)=k+\frac{z D_{z}^{\beta+k+1}\left(\Gamma(2-\alpha) z^{\alpha} D_{z}^{\alpha} f(z)\right)}{(1-\beta-k) D_{z}^{\beta+k}\left(\Gamma(2-\alpha) z^{\alpha} D_{z}^{\alpha} f(z)\right)} \quad \text { is univalent in } \mathbb{U}, \\
& \Psi(\alpha, \beta, k ; z)=\frac{z^{k+1} D_{z}^{\beta+k}\left(\Gamma(2-\alpha) z^{\alpha} D_{z}^{\alpha} f(z)\right)}{(2-\beta-k) D_{z}^{\beta+k-1}\left(\Gamma(2-\alpha) z^{\alpha} D_{z}^{\alpha} f(z)\right)} \in \mathcal{A}[1,1] \cap Q,
\end{aligned}
$$

then

$$
\frac{z q_{1}^{\prime}(z)}{q_{1}(z)} \prec \Phi(\alpha, \beta, k ; z) \prec \frac{z q_{2}^{\prime}(z)}{q_{2}(z)}
$$

implies

$$
q_{1}(z) \prec \Psi(\alpha, \beta, k ; z) \prec q_{2}(z)
$$

and $q_{1}, q_{2}$ are, respectively, the best subordinant and the best dominant.

Proof. Let $g(z)=\Theta^{\alpha, \beta} f(z)$ be defined as in Definition 1.2, where $f \in \mathcal{A}_{0}, 0<\beta<1$ and $(\alpha \neq 2,3,4, \ldots)$. Then from (1.17), we have for $k \in \mathbb{N}$,

$$
\begin{aligned}
g^{(k)}(z) & =\frac{d^{k}}{d z^{k}}\left(\Theta^{\alpha, \beta} f(z)\right) \\
& =L(2,2-\alpha)\left(\frac{d^{k}}{d z^{k}}\left(\Omega^{\beta} f(z)\right)\right) \\
& =L(2,2-\alpha) \Omega^{\beta+k} f(z) \\
& =\Theta^{\alpha, \beta+k} f(z)
\end{aligned}
$$

This yields

$$
\begin{aligned}
& \frac{z^{k} g^{(k)}(z)}{g^{(k-1)}(z)}=\frac{z^{k} \Theta^{\alpha, \beta+k} f(z)}{\Theta^{\alpha, \beta+k-1} f(z)}=\frac{z^{k+1} D_{z}^{\beta+k}\left(\Gamma(2-\alpha) z^{\alpha} D_{z}^{\alpha} f(z)\right)}{(2-\beta-k) D_{z}^{\beta+k-1}\left(\Gamma(2-\alpha) z^{\alpha} D_{z}^{\alpha} f(z)\right)}, \\
& \frac{z g^{(k+1)}(z)}{g^{(k)}(z)}=\frac{z \Theta^{\alpha, \beta+k+1} f(z)}{\Theta^{\alpha, \beta+k} f(z)}=k+\frac{z D_{z}^{\beta+k+1}\left(\Gamma(2-\alpha) z^{\alpha} D_{z}^{\alpha} f(z)\right)}{(1-\beta-k) D_{z}^{\beta+k}\left(\Gamma(2-\alpha) z^{\alpha} D_{z}^{\alpha} f(z)\right)} .
\end{aligned}
$$

By applying Lemma 2.2 for $\lambda=1$ and $\mu=0$, we get the result. 
Putting $\alpha=0$ in Theorem 2.3, we get the following corollary.

Corollary 2.4. Let $q_{1}, q_{2}$ be two nonzero univalent functions in $\mathbb{U}$, and let $k \in \mathbb{N}, 0<\beta<1$. Further, assume that $z q_{1}^{\prime}(z) / q_{1}(z)$ and $z q_{2}^{\prime}(z) / q_{2}(z)$ are starlike univalent in $\mathbb{U}$. If $f \in \mathcal{A}_{0}$,

$$
\begin{aligned}
& \Phi(\beta, k ; z)=k+\frac{z D_{z}^{\beta+k+1} f(z)}{(1-\beta-k) D_{z}^{\beta+k} f(z)} \text { is univalent in } \mathbb{U}, \\
& \Psi(\beta, k ; z)=\frac{z^{k+1} D_{z}^{\beta+k} f(z)}{(2-\beta-k) D_{z}^{\beta+k-1} f(z)} \in \mathcal{A}[1,1] \cap Q,
\end{aligned}
$$

then

$$
\frac{z q_{1}^{\prime}(z)}{q_{1}(z)} \prec \Phi(\beta, k ; z) \prec \frac{z q_{2}^{\prime}(z)}{q_{2}(z)}
$$

implies

$$
q_{1}(z) \prec \Psi(\beta, k ; z) \prec q_{2}(z)
$$

and $q_{1}, q_{2}$ are, respectively, the best subordinant and the best dominant.

In particular, for $k=1$, Corollary 2.4 reduces to the following remark.

Remark 2.5. Let $q_{1}, q_{2}$ be two nonzero univalent functions in $\mathbb{U}$, and assume that $z q_{1}^{\prime}(z) / q_{1}(z)$ and $z q_{2}^{\prime}(z) / q_{2}(z)$ are starlike univalent in $\mathbb{U}$. For $0<\beta<1$, if $f \in \mathcal{A}_{0}, 1-$ $\left(z D_{z}^{\beta+2} f(z) / \beta D_{z}^{\beta+1} f(z)\right)$ is univalent in $\mathbb{U}$ and $z^{2} D_{z}^{\beta+1} f(z) /(1-\beta) D_{z}^{\beta} f(z) \in \mathcal{A}[1,1] \cap Q$, then

$$
\frac{z q_{1}^{\prime}(z)}{q_{1}(z)} \prec 1-\frac{1}{\beta}\left(\frac{z D_{z}^{\beta+2} f(z)}{D_{z}^{\beta+1} f(z)}\right) \prec \frac{z q_{2}^{\prime}(z)}{q_{2}(z)}
$$

implies

$$
q_{1}(z) \prec \frac{1}{1-\beta}\left(\frac{z^{2} D_{z}^{\beta+1} f(z)}{D_{z}^{\beta} f(z)}\right) \prec q_{2}(z),
$$

and $q_{1}, q_{2}$ are, respectively, the best subordinant and the best dominant.

Remark 2.6. Taking $\alpha=1$ in Theorem 2.3 yields that Corollary 2.4 and Remark 2.5 are also hold true for $f(z)=z g^{\prime}(z)$, where $g \in \mathcal{A}_{0}$. 


\section{Some Properties of the Class $\mathrm{SP}_{\alpha, \beta}$}

We need the following results in our investigation of the class $\mathrm{SP}_{\alpha, \beta}$.

Lemma 3.1 (see [12]). Let $F$ and $G$ be univalent convex functions in $\mathbb{U}$. Then the Hadamard product $F * G$ is also univalent convex in $\mathbb{U}$.

Lemma 3.2 (see [13]). Let $F$ and $G$ be univalent convex functions in $\mathbb{U}$. Also let $f \prec F$ and $g \prec G$. Then $f * g \prec F * G$.

Lemma 3.3 (see [12]). Let each of the functions $f$ and $g$ be univalent starlike of order $1 / 2$. Then, for every function $F \in \mathcal{A}$,

$$
\frac{f(z) * g(z) F(z)}{f(z) * g(z)} \in \overline{\mathrm{CH}}\{F(\mathbb{U})\}, \quad(z \in \mathbb{U})
$$

where $\overline{\mathrm{CH}}$ denotes the closed convex hull.

Theorem 3.4. If $(0 \leq \mu<\alpha \leq 1)$ and $0 \leq \beta \leq 1$, then

$$
\mathrm{SP}_{\alpha, \beta} \subset \mathrm{SP}_{\mu, \beta}
$$

Proof. Let $f \in \mathrm{SP}_{\alpha, \beta}$. Then

$$
\begin{aligned}
\Theta^{\mu, \beta} f & =L(2,2-\mu) \Omega^{\beta} f=L(2-\alpha, 2-\mu) \Theta^{\alpha, \beta} f \\
& =\varphi(2-\alpha, 2-\mu ; z) * \Theta^{\alpha, \beta} f, \\
z\left(\Theta^{\mu, \beta} f\right)^{\prime} & =L(2,1) L(2-\alpha, 2-\mu) \Theta^{\alpha, \beta} f=\varphi(2-\alpha, 2-\mu ; z) *\left\{z\left(\Theta^{\alpha, \beta} f\right)^{\prime}\right\} .
\end{aligned}
$$

Also it is known that (cf. [14])

$$
\varphi(2-\alpha, 2-\mu ; z) \in S^{*}\left(\frac{1}{2}\right) .
$$

Since $R$ is a convex region, using Lemma 3.3, we get

$$
\frac{z\left(\Theta^{\mu, \beta} f\right)^{\prime}}{\Theta^{\mu, \beta} f}=\frac{\varphi(2-\alpha, 2-\mu ; z) *\left(z\left(\Theta^{\alpha, \beta} f\right)^{\prime} / \Theta^{\alpha, \beta} f\right) \Theta^{\alpha, \beta} f}{\varphi(2-\alpha, 2-\mu ; z) * \Theta^{\alpha, \beta} f} \in R .
$$

Thus, $f \in \mathrm{SP}_{\mu, \beta}$. This completes the proof of Theorem 3.4.

Corollary 3.5. Let $0<\alpha<1$ and $0 \leq \beta \leq 1$. Then

$$
\begin{gathered}
\mathrm{SP}_{1,1} \subset \mathrm{SP}_{\alpha, 1} \subset\left(\mathrm{SP}_{0,1} \equiv \mathrm{UCV}\right) \subset\left(\mathrm{SP}_{\alpha, 0} \equiv \mathrm{SP}_{\alpha}\right) \subset\left(\mathrm{SP}_{0,0} \equiv \mathrm{SP}\right) \\
\mathrm{SP}_{1, \beta} \subset \mathrm{SP}_{\alpha, \beta} \subset \mathrm{SP}_{\beta} .
\end{gathered}
$$

In particular, the functions in $\mathrm{SP}_{\alpha, \beta}$ are parabolic starlike and they are uniformly convex when $\beta=1$. 
Corollary 3.6. Let $0 \leq \lambda<\alpha \leq 1$ and $0 \leq \mu<\beta \leq 1$. Then $\mathrm{SP}_{\alpha, \beta} \subset \mathrm{SP}_{\lambda, \mu}$.

It can be verified that the Riemann map $q$ of $\mathbb{U}$ onto the region $\mathcal{R}$, satisfying $q(0)=1$ and $q^{\prime}(0)>0$, is given by

$$
\begin{aligned}
q(z) & =1+\frac{2}{\pi^{2}}\left(\log \frac{1+\sqrt{z}}{1-\sqrt{z}}\right)^{2} \\
& =1+\frac{8}{\pi^{2}} \sum_{n=1}^{\infty}\left(\frac{1}{n} \sum_{k=0}^{n-1} \frac{1}{2 k+1}\right) z^{n} \\
& =\sum_{n=0}^{\infty} B_{n} z^{n}=1+\frac{8}{\pi^{2}}\left(z+\frac{2}{3} z^{2}+\frac{23}{45} z^{3}+\frac{44}{105} z^{4}+\cdots\right), \quad(z \in \mathbb{U}) .
\end{aligned}
$$

We define the function $H$ by

$$
H(z):=\frac{1}{z}\left\{L(2,2-\alpha) L(2,2-\beta) z \exp \left(\int_{0}^{z} \frac{q(s)-1}{s} d s\right)\right\}, \quad(z \in \mathbb{U}) .
$$

Theorem 3.7. Let $\alpha, \beta \in[0,1)$ and let $H(z)$ be defined by (3.8). Then $H(z)$ is a convex univalent function. Furthermore, if $f \in \mathrm{SP}_{\alpha, \beta}$, then

$$
\frac{f(z)}{z} \prec H(z)
$$

Proof. We first note that

$$
H(z)=\frac{\varphi(2,2-\alpha ; z)}{z} * \frac{\varphi(2,2-\beta ; z)}{z} * \exp \left(\int_{0}^{z} \frac{q(s)-1}{s} d s\right), \quad(z \in \mathbb{U})
$$

where each member of the Hadamard product in (3.10) is known to be a convex univalent function (cf. $[2,14])$. Therefore, by Lemma 3.1, $H(z)$ is a univalent convex function. Next, if $f \in \mathrm{SP}_{\alpha, \lambda}$, then

$$
\frac{z\left(\Theta^{\alpha, \beta} f(z)\right)^{\prime}}{\Theta^{\alpha, \beta} f(z)} \prec q(z)
$$

Thus, there exists a function $\omega$ satisfying the Schwarz Lemma such that

$$
\frac{\Theta^{\alpha, \beta} f(z)}{z}=\exp \left(\int_{0}^{z} \frac{q(\omega(s))-1}{s} d s\right), \quad(z \in \mathbb{U})
$$

Since $q(z)-1$ is a univalent convex function, a result of [15] (see also [16, page 50]) yields

$$
\frac{\Theta^{\alpha, \beta} f(z)}{z} \prec \exp \left(\int_{0}^{z} \frac{q(s)-1}{s} d s\right)
$$


It now follows from a known result of [14, page 508, Theorem 2] that

$$
\frac{f(z)}{z} \prec H(z)
$$

The proof of Theorem 3.7 is evidently completed.

Remark 3.8. (i) Letting $\alpha$ or $\beta$ equal to zero in Theorem 3.7, we immediately obtain a subordination result due to Srivastava and Mishra (see [9]).

(ii) Taking $\alpha=1, \beta=0$ in Theorem 3.7, we get a result of [2, page 169, Theorem 3].

Theorem 3.9. Let $\alpha, \beta \in[0,1)$. If $f \in \mathrm{SP}_{\alpha, \beta}$, then

$$
\begin{aligned}
H(-r) & \leq\left|\frac{f(z)}{z}\right| \leq H(r), \quad(|z|=r), \\
\left|\arg \left(\frac{f(z)}{z}\right)\right| & \leq \max _{\theta \in[0,2 \pi]}\left\{\arg \left(H\left(r e^{i \theta}\right)\right)\right\}, \quad\left(z=r e^{i \theta}\right),
\end{aligned}
$$

where $H(z)$ is defined by (3.8). Equality holds true in (3.15) and (3.16) for some $z \neq 0$ if and only if $f$ is a rotation of $z H(z)$.

Proof. Let $f \in \mathrm{SP}_{\alpha, \beta}$. Then, by Theorem 3.7 and the Lindelöf principle of subordination, we get

$$
\begin{aligned}
\inf _{|z|=r} \operatorname{Re}\{H(z)\} & \leq \inf _{|z| \leq r} \operatorname{Re}\left\{\frac{f(z)}{z}\right\} \leq \sup _{|z| \leq r} \operatorname{Re}\left\{\frac{f(z)}{z}\right\} \\
& \leq \sup _{|z| \leq r}\left|\frac{f(z)}{z}\right| \leq \sup _{|z| \leq r} \operatorname{Re}\{H(z)\} .
\end{aligned}
$$

Since $H(z)$ is a univalent convex function and has real coefficients, $H(\mathbb{U})$ is a convex region symmetric with respect to real axis. Hence,

$$
\begin{aligned}
& \inf _{|z| \leq r} \operatorname{Re}\{H(z)\}=\inf _{-r \leq x \leq r} H(x)=H(-r), \\
& \sup _{|z| \leq r} \operatorname{Re}\{H(z)\}=\sup _{-r \leq x \leq r} H(x)=H(r) .
\end{aligned}
$$

Thus, (3.17) gives the assertion (3.15) of Theorem 3.9. Also, we readily have the assertion (3.16) of Theorem 3.9. The sharpness in (3.15) and (3.16) is also a consequence of the principle of subordination. This completes the proof of Theorem 3.9.

Corollary 3.10. Let $f \in \mathrm{SP}_{\alpha, \beta}$, where $\alpha, \beta \in[0,1]$. Then,

$$
\{w:|w| \leq H(-1)\} \subseteq f(\mathbb{U}) .
$$

The result is sharp. 
Remark 3.11. (i) Letting $\alpha$ or $\beta$ equal to zero in Theorem 3.9, we obtain a result due to Srivastava and Mishra (see [9]).

(ii) Taking $\alpha=1, \beta=0$ in Theorem 3.9, we get a result of [2, page 170, Corollary 3].

Next, we investigate characterization for $f$ to be in the class $\mathrm{SP}_{\alpha, \beta} \cap \tau$. We need first the following lemma.

Lemma 3.12. If $\Theta^{\alpha, \beta} f \in \tau$, where $\alpha, \beta \in[0,1]$, then

$$
\sum_{n=1}^{\infty} \frac{(n+1)((n+1) !)^{2}}{(2-\alpha)_{n}(2-\beta)_{n}} a_{n} \leq 1
$$

Proof. Suppose $\sum_{n=1}^{\infty}\left((n+1)((n+1) !)^{2} /(2-\alpha)_{n}(2-\beta)_{n}\right) a_{n}>1$. We can write

$$
\sum_{n=1}^{\infty} \frac{(n+1)((n+1) !)^{2}}{(2-\alpha)_{n}(2-\beta)_{n}} a_{n}=1+\varepsilon, \quad(\varepsilon>0) .
$$

Then, there exists an integer $N$ such that

$$
\sum_{n=1}^{N} \frac{(n+1)((n+1) !)^{2}}{(2-\alpha)_{n}(2-\beta)_{n}} a_{n}>1+\frac{\varepsilon}{2}
$$

For $(1 /(1+\varepsilon / 2))^{1 / N}<z<1$, we have

$$
\begin{aligned}
\left(\Theta^{\alpha, \beta} f\right)^{\prime}(z) & =1-\sum_{n=1}^{\infty} \frac{(n+1)((n+1) !)^{2}}{(2-\alpha)_{n}(2-\beta)_{n}} a_{n} z^{n} \\
& \leq 1-\sum_{n=1}^{N} \frac{(n+1)((n+1) !)^{2}}{(2-\alpha)_{n}(2-\beta)_{n}} a_{n} z^{n} \\
& \leq 1-z^{N} \sum_{n=1}^{N} \frac{(n+1)((n+1) !)^{2}}{(2-\alpha)_{n}(2-\beta)_{n}} a_{n} \\
& <1-z^{N}\left(1+\frac{\varepsilon}{2}\right) \\
& <0
\end{aligned}
$$

Since $\left(\Theta^{\alpha, \beta} f\right)^{\prime}(0)=1>0$, there exists a real number $z_{0}, 0<z_{0}<1$, such that $\left(\Theta^{\alpha, \beta} f\right)^{\prime}\left(z_{0}\right)=0$. Hence, $\Theta^{\alpha, \beta} f$ is not univalent.

Theorem 3.13. Let $\alpha, \beta \in[0,1]$. Then, a function $f \in \mathrm{SP}_{\alpha, \beta} \cap \tau$ if and only if

$$
\sum_{n=1}^{\infty} \frac{(2 n+1)((n+1) !)^{2}}{(2-\alpha)_{n}(2-\beta)_{n}} a_{n}<1
$$


Proof. First, consider

$$
\begin{aligned}
\left|\frac{z\left(\Theta^{\alpha, \beta} f\right)^{\prime}(z)}{\Theta^{\alpha, \beta} f(z)}-1\right|-\operatorname{Re}\left\{\frac{z\left(\Theta^{\alpha, \beta} f\right)^{\prime}(z)}{\Theta^{\alpha, \beta} f(z)}-1\right\} & \leq 2\left|\frac{z\left(\Theta^{\alpha, \beta} f\right)^{\prime}(z)}{\Theta^{\alpha, \beta} f(z)}-1\right| \\
& \leq \frac{\sum_{n=1}^{\infty}\left(2 n((n+1) !)^{2} /(2-\alpha)_{n}(2-\beta)_{n}\right)\left|a_{n}\right||z|^{n+1}}{1-\sum_{n=1}^{\infty}\left(((n+1) !)^{2} /(2-\alpha)_{n}(2-\beta)_{n}\right)\left|a_{n}\right||z|^{n+1}} \\
& \leq \frac{\sum_{n=1}^{\infty}\left(2 n((n+1) !)^{2} /(2-\alpha)_{n}(2-\beta)_{n}\right) a_{n}}{1-\sum_{n=1}^{\infty}\left(((n+1) !)^{2} /(2-\alpha)_{n}(2-\beta)_{n}\right) a_{n}},
\end{aligned}
$$

where $1-\sum_{n=1}^{\infty}\left(((n+1) !)^{2} /(2-\alpha)_{n}(2-\beta)_{n}\right)\left|a_{n}\right||z|^{n+1}>0$. Hence, if (3.24) holds, then the above expression is less than 1 , and consequently

$$
\left|\frac{z\left(\Theta^{\alpha, \beta} f\right)^{\prime}(z)}{\Theta^{\alpha, \beta} f(z)}-1\right|<\operatorname{Re}\left\{\frac{z\left(\Theta^{\alpha, \beta} f\right)^{\prime}(z)}{\Theta^{\alpha, \beta} f(z)}\right\}
$$

Conversely, if $f \in \mathrm{SP}_{\alpha, \beta} \cap \tau$ and $z$ is real, we get

$$
\begin{array}{r}
\frac{1-\sum_{n=1}^{\infty}\left((n+1)((n+1) !)^{2} /(2-\alpha)_{n}(2-\beta)_{n}\right) a_{n} z^{n}}{1-\sum_{n=1}^{\infty}\left(((n+1) !)^{2} /(2-\alpha)_{n}(2-\beta)_{n}\right) a_{n} z^{n}} \\
>\frac{\sum_{n=1}^{\infty}\left(n((n+1) !)^{2} /(2-\alpha)_{n}(2-\beta)_{n}\right) a_{n} z^{n}}{1-\sum_{n=1}^{\infty}\left(((n+1) !)^{2} /(2-\alpha)_{n}(2-\beta)_{n}\right) a_{n} z^{n}} .
\end{array}
$$

Let $z \rightarrow 1^{-}$along the real axis, then we get

$$
\frac{1-\sum_{n=1}^{\infty}\left((2 n+1)((n+1) !)^{2} /(2-\alpha)_{n}(2-\beta)_{n}\right) a_{n}}{1-\sum_{n=1}^{\infty}\left(((n+1) !)^{2} /(2-\alpha)_{n}(2-\beta)_{n}\right) a_{n}}>0 .
$$

Using Lemma 3.12, we have

$$
1-\sum_{n=1}^{\infty} \frac{((n+1) !)^{2}}{(2-\alpha)_{n}(2-\beta)_{n}} a_{n}>1-\sum_{n=1}^{\infty} \frac{(n+1)((n+1) !)^{2}}{(2-\alpha)_{n}(2-\beta)_{n}} a_{n} \geq 0 .
$$

Therefore, the denominator in (3.28) is positive, and hence (3.24) holds. This completes the proof of Theorem 3.13.

Remark 3.14. Theorem 3.13 is sharp for functions of the form

$$
\Theta^{\alpha, \beta} f_{n}(z)=z-\frac{(2-\alpha)_{n}(2-\beta)_{n}}{(2 n+1)((n+1) !)^{2}} z^{n+1}, \quad(n \geq 1) .
$$


Corollary 3.15. If $\alpha \in[0,1]$, then

$$
f \in \mathrm{SP}_{\alpha} \cap \tau \Longleftrightarrow \sum_{n=1}^{\infty} \frac{(n+1) !(2 n+1)}{(2-\alpha)_{n}} a_{n}<1
$$

In particular, $f \in \mathrm{SP} \cap \tau$ if and only if $\sum_{n=1}^{\infty}(2 n+1) a_{n}<1$ and $f \in \mathrm{UCV} \cap \tau$ if and only if $\sum_{n=1}^{\infty}(2 n+1)(n+1) a_{n}<1$.

Corollary 3.16. If $f \in \mathrm{SP}_{\alpha, \beta} \cap \tau$, where $\alpha, \beta \in[0,1]$, then

$$
a_{n} \leq \frac{(2-\alpha)_{n}(2-\beta)_{n}}{(2 n+1)((n+1) !)^{2}}, \quad(n \geq 1) .
$$

Corollary 3.17. If $f \in \mathrm{SP}_{\alpha, \beta} \cap \tau$, where $\alpha, \beta \in[0,1]$, then

$$
|z|-\frac{(2-\alpha)(2-\beta)}{12}|z|^{2}<|f(z)|<|z|+\frac{(2-\alpha)(2-\beta)}{12}|z|^{2}, \quad(z \in \mathbb{U}) .
$$

Proof. Let $f \in \mathrm{SP}_{\alpha, \beta} \cap \tau$, where $\alpha, \beta \in[0,1]$. Clearly,

$$
\frac{12}{(2-\alpha)(2-\beta)} \sum_{n=1}^{\infty} a_{n} \leq \sum_{n=1}^{\infty} \frac{(2 n+1)((n+1) !)^{2}}{(2-\alpha)_{n}(2-\beta)_{n}} a_{n}<1 .
$$

Therefore,

$$
\begin{aligned}
& |f(z)| \geq|z|-|z|^{2} \sum_{n=1}^{\infty} a_{n}>|z|-\frac{(2-\alpha)(2-\beta)}{12}|z|^{2}, \\
& |f(z)| \leq|z|+|z|^{2} \sum_{n=1}^{\infty} a_{n}<|z|-\frac{(2-\alpha)(2-\beta)}{12}|z|^{2} .
\end{aligned}
$$

Remark 3.18. Under the hypothesis of Corollary 3.17, $f(z)$ lies in a disc centered at the origin with radius $\mathbf{r}$ given by

$$
\mathbf{r}=1+\frac{(2-\alpha)(2-\beta)}{12}
$$

In particular, we have

(i) if $f \in \mathrm{SP} \cap \tau$, then $f(z)$ lies in a disc centered at the origin with radius 4/3;

(ii) if $f \in \mathrm{SP}_{\alpha} \cap \tau$, then $f(z)$ lies in a disc centered at the origin with radius $(8-\alpha) / 6$;

(iii) if $f \in \mathrm{UCV} \cap \tau$, then $f(z)$ lies in a disc centered at the origin with radius $7 / 6$;

(iv) if $f \in \mathrm{SP}_{1,1} \cap \tau$, then $f(z)$ lies in a disc centered at the origin with radius 13/12. 
Consequently, let $\Theta^{\alpha_{1}, \alpha_{2}, \ldots, \alpha_{k}}$ be defined by

$$
\Theta^{\alpha_{1}, \alpha_{2}, \ldots, \alpha_{k}} f(z)=\left(\Omega^{\alpha_{1}} \circ \Omega^{\alpha_{2}} \circ \cdots \circ \Omega^{\alpha_{k}}\right) f(z), \quad \alpha_{j} \in[0,1] .
$$

Then, $f$ belonging to the corresponding class $\mathrm{SP}_{\underbrace{1,1, \ldots, 1}_{k \text {-imes }}}^{1,} \cap$ implies $f(z)$ lies in a disc centered at the origin with radius $\mathbf{r}_{k}$ given by

$$
\mathbf{r}_{k}=1+\frac{1}{3 \times 2^{k}}
$$

\section{Class-Preserving Operators and Transforms}

Theorem 4.1. Let $\Theta^{\lambda, \beta} f$ be univalent starlike function of order $1 / 2\left(f \in \mathcal{A}_{0}\right)$ and let $g \in \mathrm{SP}_{\alpha, \beta}(\lambda \leq$ $\alpha)$. Then,

$$
\Theta^{\lambda, \beta} f * \Theta^{\alpha, \beta} g \in \mathrm{SP}_{\alpha, \beta}
$$

In particular, if $\Theta^{\alpha, \beta} f$ is univalent starlike function of order $1 / 2$ and $g \in \mathrm{SP}_{\alpha, \beta}$, then

$$
\Theta^{\alpha, \beta} f * \Theta^{\alpha, \beta} g \in \mathrm{SP}_{\alpha, \beta}
$$

Proof. Let $\Theta^{\lambda, \beta} f$ be univalent starlike function of order $1 / 2\left(f \in \mathcal{A}_{0}\right)$ and $g \in \operatorname{SP}_{\alpha, \beta}(\lambda \leq \alpha)$. By definition,

$$
f \in S^{*}\left(\frac{1}{2}\right), \quad \Theta^{\alpha, \beta} g \in \mathrm{SP} \subset S^{*}\left(\frac{1}{2}\right) .
$$

The commutative and associative properties of the Hadamard product yield

$$
\begin{aligned}
z\left(\Theta^{\Lambda, \beta} f * \Theta^{\alpha, \beta} g\right)^{\prime} & =L(2,1)\left(\Theta^{\Lambda, \beta} f * \Theta^{\alpha, \beta} g\right) \\
& =\Theta^{\lambda, \beta} f * L(2,1) \Theta^{\alpha, \beta} g \\
& =\Theta^{\lambda, \beta} f *\left\{z\left(\Theta^{\alpha, \beta} g\right)^{\prime}\right\} .
\end{aligned}
$$

Therefore, using Lemma 3.3, we get

$$
\frac{z\left(\Theta^{\lambda, \beta} f * \Theta^{\alpha, \beta} g\right)^{\prime}}{\Theta^{\lambda, \beta} f * \Theta^{\alpha, \beta} g}=\frac{\Theta^{\lambda, \beta} f *\left(\left(z\left(\Theta^{\alpha, \beta} g\right)^{\prime} / \Theta^{\alpha, \beta} g\right) \Theta^{\alpha, \beta} g\right)}{\Theta^{\lambda, \beta} f * \Theta^{\alpha, \beta} g} \in \mathcal{R}, \quad(z \in \mathbb{U}) .
$$

This completes the proof of Theorem 4.1. 
Taking $\alpha=1$ and $\beta=0$ in Theorem 4.1, then we have the following corollary.

Corollary 4.2. If $\Omega^{\lambda} f(z) \in S^{*}(1 / 2)$ and $g(z) \in \mathrm{UCV}$, then $\Omega^{\lambda} f(z) * z g^{\prime}(z) \in \mathrm{UCV}$. In particular, if $\Omega^{\curlywedge} f(z) \in \mathrm{SP}$ and $g(z) \in \mathrm{UCV}$, then $\Omega^{\lambda} f(z) * z g^{\prime}(z) \in \mathrm{UCV}$. Moreover, if $\Omega^{\curlywedge} f(z) \in \mathrm{UCV}$ and $g(z) \in \mathrm{UCV}$, then $\Omega^{\curlywedge} f(z) * z g^{\prime}(z) \in \mathrm{UCV}$.

Taking $\lambda=0$ or $1, \alpha=1$, and $\beta=0$ in Theorem 4.1 , then we have the following corollary.

Corollary 4.3. If $f(z) \in S^{*}(1 / 2)$ and $g(z) \in \mathrm{UCV}$, then $f(z) * z g^{\prime}(z) \in \mathrm{UCV}$. In particular, if $f(z) \in \mathrm{SP}$ and $g(z) \in \mathrm{UCV}$, then $f(z) * z g^{\prime}(z) \in \mathrm{UCV}$. Moreover, if $f(z) \in \mathrm{UCV}$ and $g(z) \in \mathrm{UCV}$, then $f(z) * z g^{\prime}(z) \in \mathrm{UCV}$.

Corollary 4.4 (see [9]). If $\Omega^{\lambda} f \in S^{*}(1 / 2)$ and $g \in \mathrm{SP}_{\mu}(\lambda \leq \mu)$, then

$$
\Omega^{\lambda} f * \Omega^{\mu} g \in \mathrm{SP}_{\mu}
$$

In particular, if $\Omega^{\curlywedge} f \in S^{*}(1 / 2)$ and $g \in \mathrm{SP}_{\lambda}$, then

$$
\Omega^{\lambda} f * \Omega^{\lambda} g \in \mathrm{SP}_{\curlywedge}
$$

Corollary 4.5 (see [3]). If $f \in S^{*}(1 / 2)$ and $g \in \mathrm{SP}$, then $f * g \in \mathrm{SP}$. In particular, if $f \in \mathrm{SP}$ and $g \in \mathrm{SP}$, then $f * g \in \mathrm{SP}$.

Theorem 4.6. Let $f \in \mathrm{SP}_{\beta}$ and $g \in \mathrm{SP}_{\alpha, \beta}$, where $\alpha, \beta \in[0,1]$. Then, $f * g \in \mathrm{SP}_{\alpha, \beta}$.

Proof. The proof of Theorem 4.6 is similar to that of Theorem 4.1. Let $f \in \mathrm{SP}_{\beta}$ and $g \in \mathrm{SP}_{\alpha, \beta}$. We first note that

$$
\begin{gathered}
z\left(\Theta^{\alpha, \beta}(f * g)(z)\right)^{\prime}=\Omega^{\alpha} g(z) * z\left(\Omega^{\beta} f(z)\right)^{\prime}, \\
\Theta^{\alpha, \beta}(f * g)(z)=\Omega^{\alpha} g(z) * \Omega^{\beta} f(z) .
\end{gathered}
$$

Therefore, using Lemma 3.3, we get

$$
\frac{z\left(\Theta^{\alpha, \beta}(f * g)(z)\right)^{\prime}}{\Theta^{\alpha, \beta}(f * g)(z)}=\frac{\Omega^{\alpha} g(z) *\left(z\left(\Omega^{\beta} f(z)\right)^{\prime} / \Omega^{\beta} f(z)\right) \Omega^{\beta} f(z)}{\Omega^{\alpha} g(z) * \Omega^{\beta} f(z)} \in \mathcal{R}, \quad(z \in \mathbb{U}) .
$$

Thus, $f * g \in \mathrm{SP}_{\alpha, \beta}$. This completes the proof of Theorem 4.6.

Corollary 4.7. The class $\mathrm{SP}_{\alpha}(0 \leq \alpha \leq 1)$ is closed under convolution, and in particular the classes $\mathrm{SP}$ and $\mathrm{UCV}$ are so.

Theorem 4.8. Let $f \in \mathrm{SP}_{1, \beta}(0 \leq \beta \leq 1)$ and $g \in \mathrm{SP}_{\alpha, \beta}(0 \leq \alpha \leq 1)$. Then, $f * g \in \mathrm{SP}_{1, \beta}$. In particular, the class $\mathrm{SP}_{1, \beta}(0 \leq \beta \leq 1)$ is closed under convolution. 
Proof. The proof of Theorem 4.8 is similar to that of Theorem 4.6. Let $f \in \mathrm{SP}_{1, \beta}$ and $g \in \mathrm{SP}_{\alpha, \beta}$. We first note that

$$
\begin{gathered}
z\left(\Theta^{1, \beta}(f * g)(z)\right)^{\prime}=z f^{\prime}(z) * z\left(\Omega^{\beta} g(z)\right)^{\prime}, \\
\Theta^{1, \beta}(f * g)(z)=z f^{\prime}(z) * \Omega^{\beta} g(z) .
\end{gathered}
$$

Therefore, using Lemma 3.3, we get

$$
\frac{z\left(\Theta^{1, \beta}(f * g)(z)\right)^{\prime}}{\Theta^{1, \beta}(f * g)(z)}=\frac{z f^{\prime}(z) *\left(z\left(\Omega^{\beta} g(z)\right)^{\prime} / \Omega^{\beta} g(z)\right) \Omega^{\beta} g(z)}{z f^{\prime}(z) * \Omega^{\beta} g(z)} \in \mathcal{R}, \quad(z \in \mathbb{U}) .
$$

Thus, $f * g \in \mathrm{SP}_{1, \beta}$. By taking $\alpha=1$, we see that the class $\mathrm{SP}_{1, \beta}(0 \leq \beta \leq 1)$ is closed under convolution. This completes the proof of Theorem 4.8.

Theorem 4.9. Let $f_{j} \in \mathrm{SP}_{\alpha, \beta}(j=1,2, \ldots, n)$. Also let

$$
\mu_{j}>0, \quad \sum_{j=1}^{n} \mu_{j}=1
$$

Define a function $g$ by

$$
\Theta^{\alpha, \beta} g=\prod_{j=1}^{n}\left(\Theta^{\alpha, \beta} f_{j}\right)^{\mu_{j}}
$$

Then, $g \in \mathrm{SP}_{\alpha, \beta}$.

Proof. Let $f_{j} \in \mathrm{SP}_{\alpha, \beta}(j=1,2, \ldots, n)$ and let $g$ be defined by (4.13). Direct calculation gives

$$
\begin{aligned}
\left|\frac{z\left(\Theta^{\alpha, \beta} g\right)^{\prime}}{\Theta^{\alpha, \beta} g}-1\right| & =\left|\sum_{j=1}^{n} \mu_{j} \frac{z\left(\Theta^{\alpha, \beta} f_{j}\right)^{\prime}}{\Theta^{\alpha, \beta} f_{j}}-1\right| \\
& <\sum_{j=1}^{n} \mu_{j} \operatorname{Re}\left(\frac{z\left(\Theta^{\alpha, \beta} f_{j}\right)^{\prime}}{\Theta^{\alpha, \beta} f_{j}}\right) \\
& =\operatorname{Re}\left(\frac{z\left(\Theta^{\alpha, \beta} g\right)^{\prime}}{\Theta^{\alpha, \beta} g}\right) .
\end{aligned}
$$

Thus, by Definition 1.3, $g \in \mathrm{SP}_{\alpha, \beta}$. This completes the proof of Theorem 4.9.

Theorem 4.10. Let $f \in \mathrm{SP}_{\alpha, \beta}$, where $\alpha, \beta \in[0,1]$. Then, the function $F(z)$ defined by the integral transform

$$
F(z):=\frac{c+1}{z^{c}} \int_{0}^{z} t^{c-1} f(t) d t, \quad(z \in \mathbb{U} ; c>-1)
$$

is also in the class $\mathrm{SP}_{\alpha, \beta}$. 
Proof. We begin by noting that

$$
\begin{aligned}
F(z) & =L(c+1, c+2) f(z), \\
z\left(\Theta^{\alpha, \beta} F(z)\right)^{\prime} & =L(2,1) L(2,2-\alpha) L(2,2-\beta) L(c+1, c+2) f(z) \\
& =L(c+1, c+2)\left(z\left(\Theta^{\alpha, \beta} f(z)\right)^{\prime}\right) \\
& =\varphi(c+1, c+2 ; z) *\left(z\left(\Theta^{\alpha, \beta} f(z)\right)^{\prime}\right) .
\end{aligned}
$$

Using a result of Bernardi [17], it can be verified that

$$
\varphi(c+1, c+2 ; z) \in S^{*}\left(\frac{1}{2}\right)
$$

Also, by hypothesis, $\Theta^{\alpha, \beta} f(z) \in \mathrm{SP} \subset S^{*}(1 / 2)$. Thus, using Lemma 3.3, we get

$$
\frac{z\left(\Theta^{\alpha, \beta} F(z)\right)^{\prime}}{\Theta^{\alpha, \beta} F(z)}=\frac{\varphi(c+1, c+2 ; z) *\left(z\left(\Theta^{\alpha, \beta} f(z)\right)^{\prime} / \Theta^{\alpha, \beta} f(z)\right) \Theta^{\alpha, \beta} f(z)}{\varphi(c+1, c+2 ; z) * \Theta^{\alpha, \beta} f(z)} \in \mathcal{R},
$$

which completes the proof of Theorem 4.10.

\section{Acknowledgment}

The work presented here was supported by EScienceFund 04-01-02-SF0425, Academy of Sciences, Malaysia.

\section{References}

[1] A. W. Goodman, “On uniformly convex functions," Annales Polonici Mathematici, vol. 56, no. 1, pp. 87-92, 1991.

[2] W. C. Ma and D. Minda, “Uniformly convex functions," Annales Polonici Mathematici, vol. 57, no. 2, pp. 165-175, 1992.

[3] F. Rønning, "Uniformly convex functions and a corresponding class of starlike functions," Proceedings of the American Mathematical Society, vol. 118, no. 1, pp. 189-196, 1993.

[4] B. C. Carlson and D. B. Shaffer, "Starlike and prestarlike hypergeometric functions," SIAM Journal on Mathematical Analysis, vol. 15, no. 4, pp. 737-745, 1984.

[5] S. Owa and H. M. Srivastava, "Univalent and starlike generalized hypergeometric functions," Canadian Journal of Mathematics, vol. 39, no. 5, pp. 1057-1077, 1987.

[6] S. Owa, "On the distortion theorems-I," Kyungpook Mathematical Journal, vol. 18, no. 1, pp. 53-59, 1978.

[7] H. M. Srivastava and S. Owa, "An application of the fractional derivative," Mathematica Japonica, vol. 29 , no. 3, pp. 383-389, 1984.

[8] H. M. Srivastava and S. Owa, Eds., Univalent Functions, Fractional Calculus, and Their Applications, Ellis Horwood Series: Mathematics and Its Applications, Halsted Press/John Wiley \& Sons, New York, NY, USA, 1989

[9] H. M. Srivastava and A. K. Mishra, "Applications of fractional calculus to parabolic starlike and uniformly convex functions," Computers \& Mathematics with Applications, vol. 39, no. 3-4, pp. 57-69, 2000.

[10] S. S. Miller and P. T. Mocanu, "Subordinants of differential superordinations," Complex Variables: Theory and Application, vol. 48, no. 10, pp. 815-826, 2003. 
[11] O. Al-Refai and M. Darus, "Main differential sandwich theorem with some applications," Lobachevskii Journal of Mathematics, in press, 2009.

[12] St. Ruscheweyh and T. Sheil-Small, "Hadamard products of Schlicht functions and the PólyaSchoenberg conjecture," Commentarii Mathematici Helvetici, vol. 48, no. 1, pp. 119-135, 1973.

[13] St. Ruscheweyh and J. Stankiewicz, "Subordination under convex univalent functions," Bulletin of the Polish Academy of Sciences, Mathematics, vol. 33, no. 9-10, pp. 499-502, 1985.

[14] Y. Ling and S. Ding, "A class of analytic functions defined by fractional derivation," Journal of Mathematical Analysis and Applications, vol. 186, no. 2, pp. 504-513, 1994.

[15] G. M. Goluzin, "On the majorization principle in function theory," Doklady Akademii Nauk SSSR, vol. 42, pp. 647-650, 1935 (Russian).

[16] Ch. Pommerenke, Univalent Functions, Vandenhoeck \& Ruprecht, Göttingen, Germany, 1975.

[17] S. D. Bernardi, "Convex and starlike univalent functions," Transactions of the American Mathematical Society, vol. 135, pp. 429-446, 1969. 


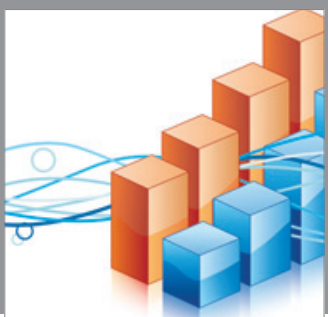

Advances in

Operations Research

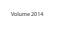

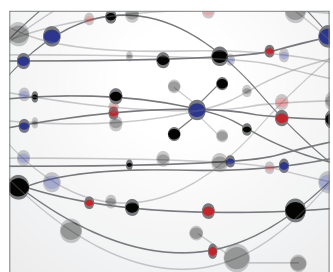

\section{The Scientific} World Journal
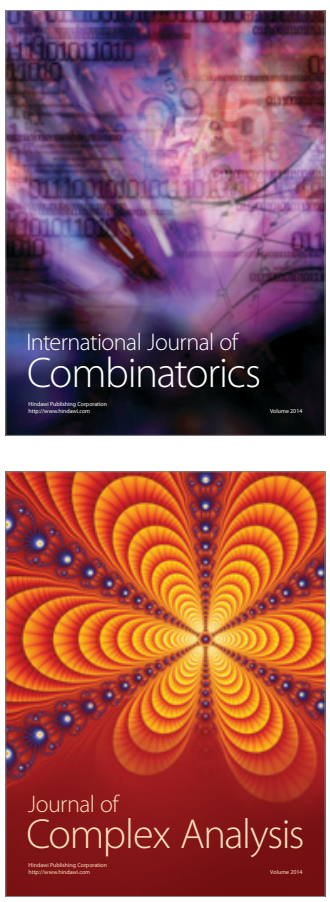

International Journal of

Mathematics and

Mathematical

Sciences
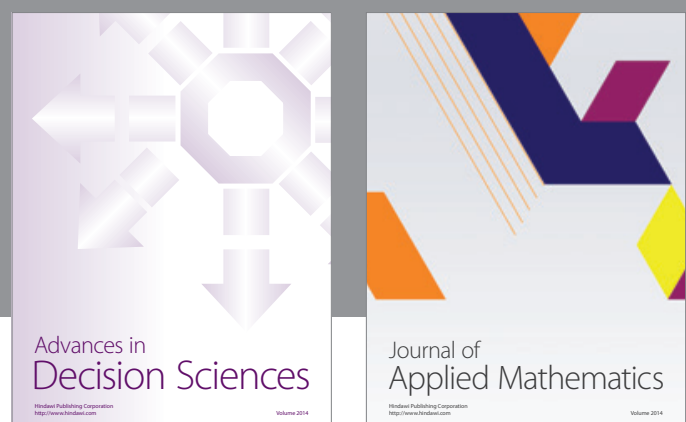

Journal of

Applied Mathematics
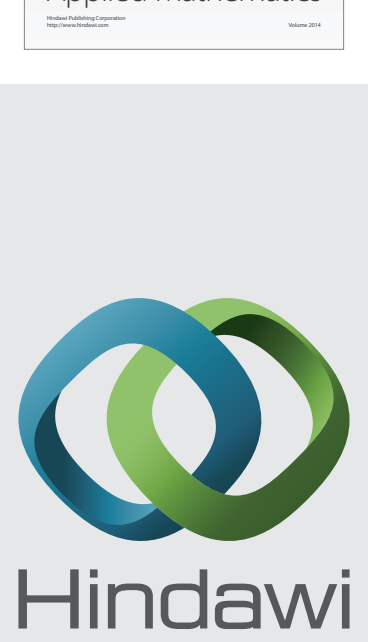

Submit your manuscripts at http://www.hindawi.com
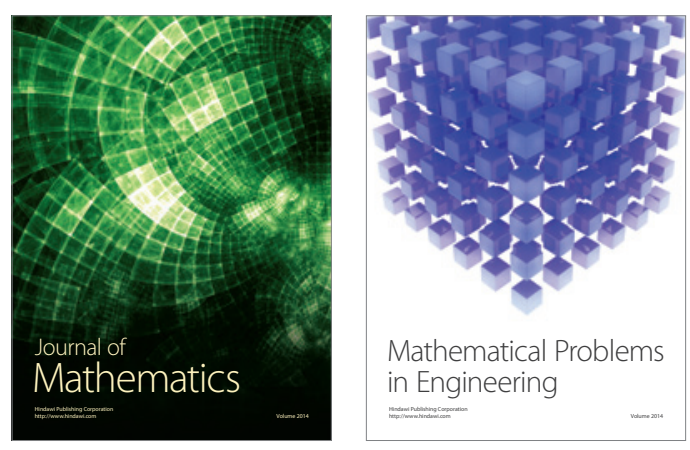

Mathematical Problems in Engineering
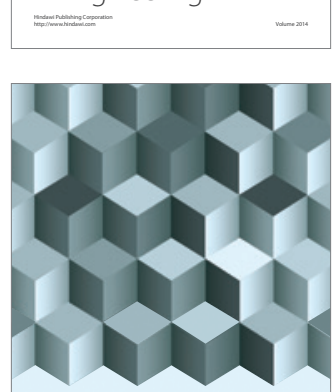

Journal of

Function Spaces
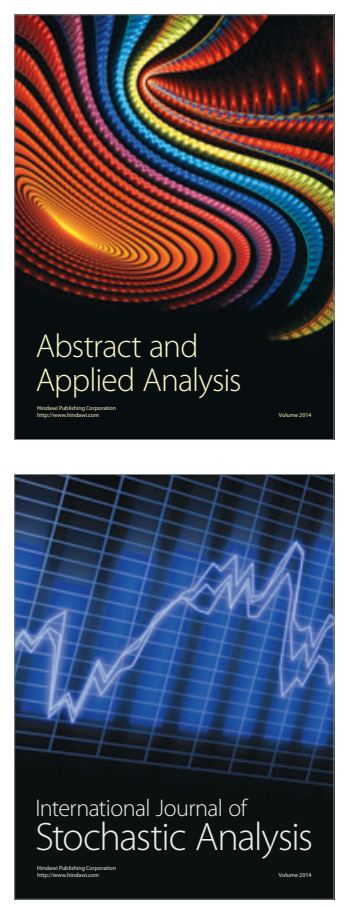

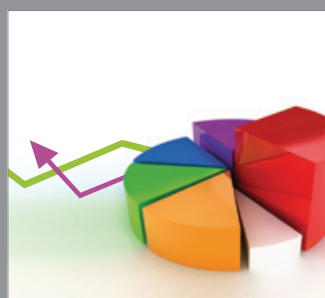

ournal of

Probability and Statistics

Promensencen
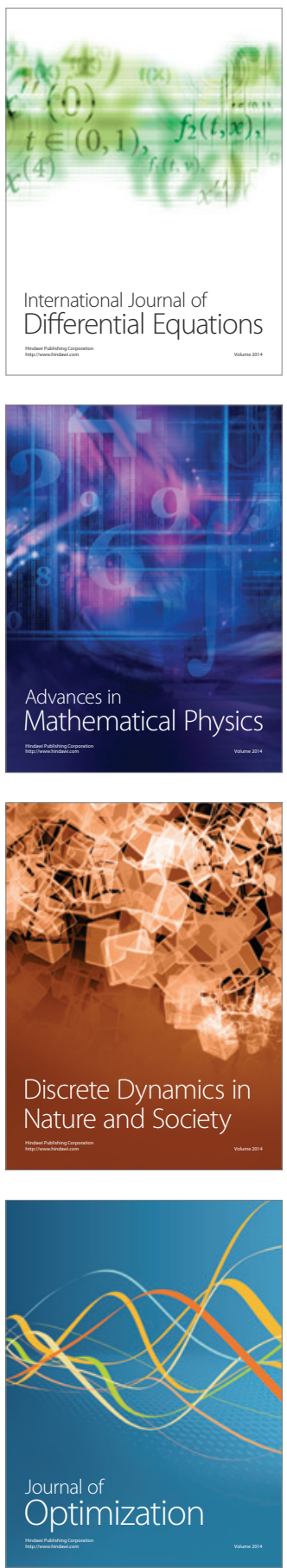